\title{
General Dual Wiimote 3D Localization Scheme: Sensitivity Analysis and Its Application on Robot Arm Attitude Detection
}

\author{
Sing-Chi Hsu*, Ting-Hao Li, Hui-Yuan Chan, and Kuo-Shen Chen \\ Department of Mechanical Engineering, National Cheng Kung University, \\ No. 1, Dasyue Rd., East Dist., Tainan City 701, Taiwan \\ *Corresponding Author: singchihsu@gmail.com
}

\begin{abstract}
Indoor localization has been receiving great interest and 2D localization system based on infrared LED-based wiimote camera is also well-developed. Although 3D positioning techniques based on infrared LED detection have been proposed and validated, it is not yet matured for general indoor localization due to its insufficient sensing zone and specific layout attitude, which may not always been satisfied. In this work, based on our previous research, we propose a layout to extend the sensing region to cover the entire indoor space by deploying many wiimote 3D pairs and make sure at least the object can be detected by at least two wiimote pairs during motion. However, this also implies that the original optimal deployed attitude cannot be maintained. As a result, to deal with this problem, a variable-angle dual wiimote $3 \mathrm{D}$ system is developed and corresponding localization performance and sensitivity analysis is carried out for clarifying the above concern. From sensitivity analysis result, deployment of wiimote cameras requires higher accuracy in orientation when angles between coordinates of both cameras are $90^{\circ}$, and tolerance in position error decreases when angles between both cameras is further from $90^{\circ}$. Furthermore, the scheme is also applied to robot arm attitude determination, and shows the high possibility for this system developing into an effective external sensor for robot arm controlling. It is expected that this wiimote $3 \mathrm{D}$ localization system to have a great potential on inexpensive and precise general indoor localization system.
\end{abstract}

Keywords: Wiimote, indoor localization, robustness analysis, 3D positioning, robot attitude determination

\section{Introduction}

Indoor localization becomes increasingly important due to the development of intelligent living and building automation technologies, where accurate position information is a fundamental task on indoor mobile robot navigation ${ }^{(1)}$. Navigation, control coordinated motion, and subsequent tasks of mobile robot could be achieved with the position information. Several well-known indoor localization scheme, for instance, inertial units ${ }^{(2)}$, ultrasonic ${ }^{(3)}$, and vision cameras $^{(4)}$, have already shown their performance. However, due to restrictions of position timing, position accuracy, and complex indoor environments, a flawless positioning technique has not yet been achieved.

IR indoor positioning, which determines the location by projecting and receiving a modulation IR line with optical sensor devices, such as wiimote CMOS cameras, is another approach. This approach can be relatively accurate in 2D indoor localization applications and it already demonstrated its performance in various applications ${ }^{(6)}$. Li et al developed 3D localization scheme based on dual wiimote layout. This scheme contains one pair of wiimote cameras, which is assembled based on the CMOS camera built in Wii Remote. The 2D position of infrared LED is captured by the cameras in the form of pixel coordinates, and transformed into visual angle corresponding to the CMOS camera.

Although the feasibility has been demonstrated, in comparison with the wiimote $2 \mathrm{D}$ localization, this wiimote $3 \mathrm{D}$ localization scheme ${ }^{(7,8)}$ shows inferior performance in both the available sensing zone and the achievable accuracy in detection.

Zhan et $\mathrm{al}^{(9)}$ further utilized dual wiimote $3 \mathrm{D}$ to improve the sensing resolution. The scheme, called as the fixed-angle dual wiimote 3D localization, consists of placing two wiimote 3D 
pairs in opposite direction, the sensing resolution can be improved. The function of the additional pair of wiimote cameras is basically to offer another set of position data to compensate the inaccurate data of the original pair of wiimote cameras. Based on this investigation, a scenario is drawn to localize mobile units in entire indoor space is drawn in Figure 1. That is, by deploying many wiimote $3 \mathrm{D}$ modules to guarantee a moving object is seen at least by two wiimote 3D module simultaneously, the sensing zone can be expanded. However, this also implies that the specific optimized layout proposed by Chan cannot be met in this case. The major concern of the newly proposed scheme, called as variable-angle dual wiimote $3 \mathrm{D}$ localization, is therefore the sensing performance against the layout geometric parameters. Therefore, the performance variations with different layout parameters should be investigated. By evaluating the sensitivity of the wiimote 3D scheme against deployment attitude variations, it is possible to optimize the sensor layout inside the workspace.

In this work, both analysis and experiments are carried out to address the above sensor robustness concerns for the variable-angle dual wiimote 3D localization scheme, via both simulation and experiments. Two geometric variables, distance and orientation between coordinates of wiimote cameras, are mainly the key parameters focused in this paper. Furthermore, experimental results are then compared with the simulation results for the purpose of validation. Finally, after validation, a robot arm with several IR LEDs are then served as a platform for demonstrating the effectiveness of the variable-angle dual wiimote $3 \mathrm{D}$ localization by detecting the attitude of the robot as the first step to be integrated as an external position sensor for robot control.

The rest of the paper presents the technical detail of the development of wiimote 3D localization. In section II, the background on wiimote 3D localization is briefly reviewed for providing the background of this work. Section III describes the robustness analysis and robot arm attitude detection experiments, which are verification of the schemes mentioned in section II. Consequently, discussion and conclusion are made in section IV.

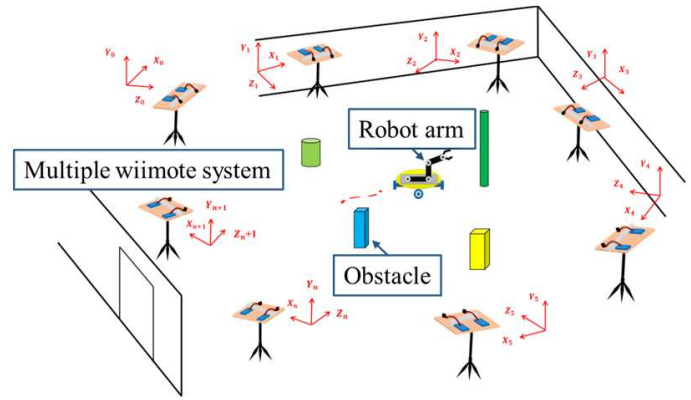

Fig. 1. Wiimote 3D localization scenario for cover the entire living space $^{(9)}$

\section{Wiimote 3D Localization System}

Wiimote 3D localization system developed from single wiimote $3 \mathrm{D}$ localization system, which contains only one pair of wiimote cameras $^{(7,8)}$, fixed-angle dual wiimote camera system, with an additional pair of wiimote cameras for data compensation, and to variable-angle dual wiimote 3D localization system, which allows more flexible deployment. These systems will be described in the following sections.

\subsection{Single Wiimote 3D Localization System}

Based on the successful on wiimote 2D localization, 3D localization based on wiimote was proposed and validated by $\mathrm{Li}$ and Chen ${ }^{(7,8)}$ using a pair of wiimote CMOS camera in a stereo vision fashion. Schematically shown in Figure 2, with the known distance between the two CMOS cameras, the pixel coordinates detected by these cameras are eventually converted to the physical location of the detected IR LEDs based on geometry and kinematics. However, since the visible angle of a single CMOS camera is $32^{\circ}$ in vertical and $42^{\circ}$ in horizontal directions, which is already not big enough, the valid zone for wiimote $3 \mathrm{D}$ localization is limited on the intersections of detected zones from both cameras. As a result, the sensing zone of wiimote $3 \mathrm{D}$ is actually much smaller than that in 2D localization. The scheme has successful demonstrated its applicability on determining the attitude of a four-bar linkage mechanism and a self-made robot arm. However, since the pixel resolution is actually distance dependent, the performance of this scheme quickly became worse if the distance between the IR LED and the CMOS camera pair exceeds $1.5 \mathrm{~m}$.

To demonstrate the distance-dependent localization error, a simple experiment is carried out. As shown in Figure 3 , infrared LED is placed 80 centimeter away from wiimote cameras and is moved in an additional 80 centimeter away from its original position in $\mathrm{Z}$ direction and moves back and forth in $\mathrm{X}$ direction in the range of negative 10 centimeter and positive 10 centimeter. Comparison between the

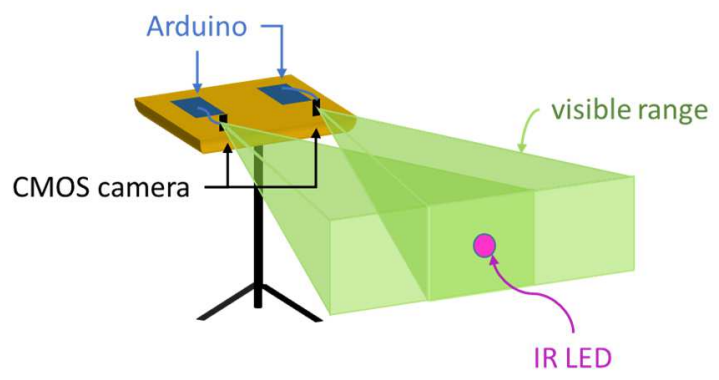

Fig. 2. Single wiimote 3D localization system 
captured 3D position of the infrared LED and the real infrared LED position is shown in Figure 3. Difference between these sets of data increased while the infrared LED moves away from the wiimote cameras. To improve this disadvantage, Chan et al proposed a scheme to use two wiimote pairs and placed in opposite direction to reduce the spatial dependent error. Such fixed-angle dual wiimote 3D localization scheme has also demonstrated its applicability elsewhere ${ }^{(9)}$ and a brief test is performed below.

\subsection{Fixed-Angle Dual Wiimote 3D Localization System}

As mentioned above, an additional pair of wiimote cameras, hereinafter referred to as" wiimote camera 2", is placed on the opposite side to the original pair of wiimote cameras, hereinafter referred to as "wiimote camera 1", shown as Figure 4. While the $\mathrm{Z}$ direction distance between the infrared LED and wiimote camera 1 is detected to be further than the distance between infrared LED and wiimote camera 2, the system will take the 3D position data captured by the wiimote camera 2 as a compensation ${ }^{(9)}$ and the data selection depends on certain criteria such as the RMS of data fluctuation. Experiment results, as shown in Figure 5, show that this system is capable to improve the inaccuracy problem of single wiimote 3D localization scheme. By this we learned that the localization accuracy can be improved using multiple wiimote 3D pairs. The CMOS cameras are very cheap and it is no problem to deploy as much as possible in a living space such that a moving object can always be seen by at least two wiimote 3D pairs. However, in practical sense, it is virtually impossible to fix the arrangement of the dual wiimote $3 \mathrm{D}$ pair in a fixed angle sense in any times. As a result, a variable-angle dual wiimote $3 \mathrm{D}$ localization scheme is developed to enhance the flexibility in wiimote camera setup to increase the adaptively for using in various indoor environments.

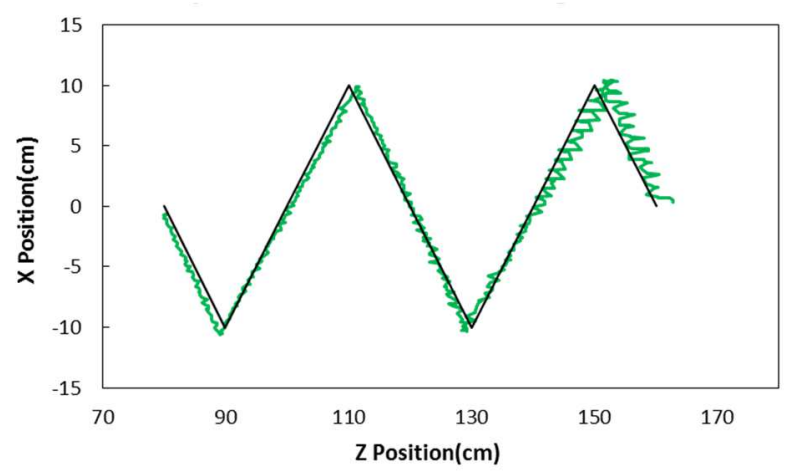

Fig. 3. Comparison of observed data by single wiimote 3D localization system and real position of infrared $\operatorname{LED}^{(9)}$

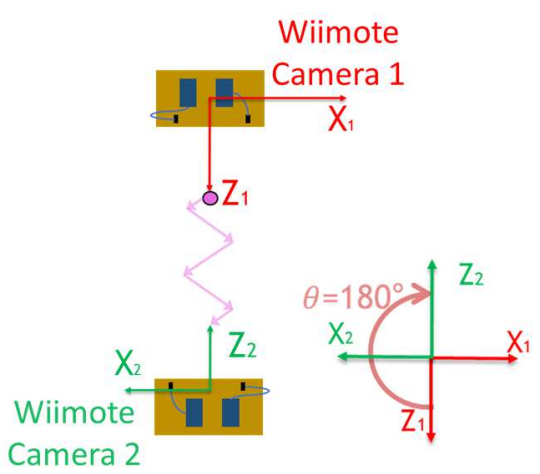

Fig. 4. Fixed-angle dual wiimote 3D localization system

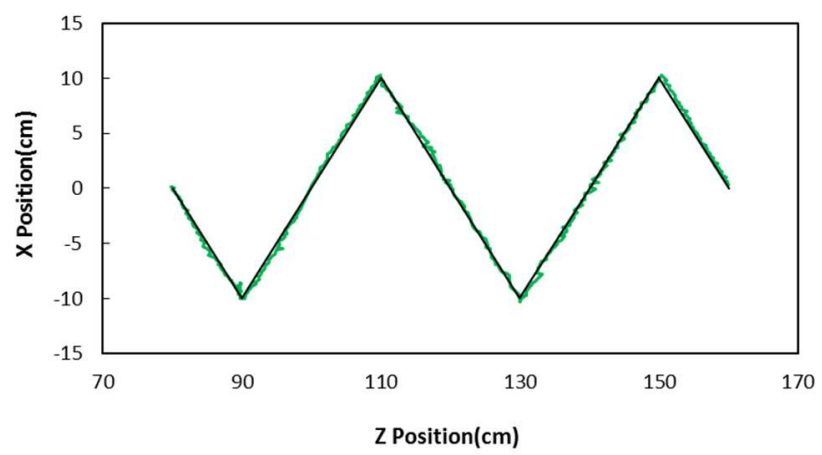

Fig. 5. Comparison of observed data by fixed-angle dual wiimote $3 \mathrm{D}$ localization system and real position of infrared $\operatorname{LED}^{(9)}$

\subsection{Variable-Angle Dual Wiimote 3D Localization System}

As addressed above, the ultimate goal of this research is to build up a general large area indoor localization system such that the moving object can be seen at least two wiimote 3D pairs at any locations and any moments, including passing certain shielding location where the object may not be observed by some wiimotes. However, this implies that the configuration of the dual wiimote $3 \mathrm{D}$ cannot have an optimized fixed angle just like Zhan's improvement (9). As a result, the sensitivity or the robustness of the dual wiimote localization scheme against is important. If the localization performance is not too sensitive to the deploy angle, the whole indoor localization would be more consistent. On the other hand, if the performance is too sensitive to the deploy angle, special care should be prepared for maintaining a consisted performance.

In order to address the concern shown right above, a test system with adjustable deploy angle and distance of wiimote $3 \mathrm{D}$ module is designed to perform the sensitivity analysis. The system is schematically shown in Figure 6. The object, 


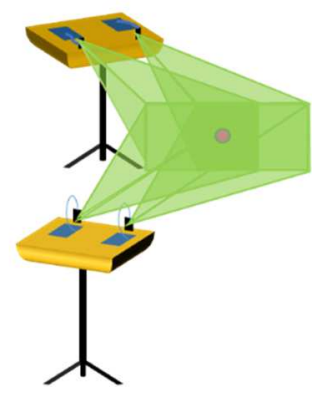

(a) 3D Illustration

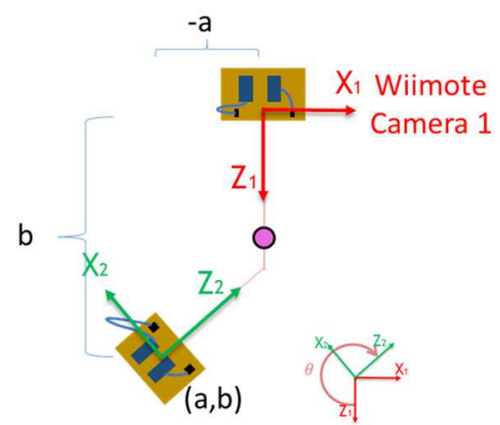

Wiimote

Camera 2

(b) 2D Illustration with coordinate definition

Fig. 6. Variable-angle dual wiimote 3D localization system illustration

containing an IR LED, will be mounted on a linear motor with known position to serve as the standard. Wiimote camera 1 is deployed in the same position as previous setup (at world coordinate $(0,0)$ and the orientation is in $\mathrm{Z}$ direction) but the wiimote position and orientation of wiimote camera 2 can be adjusted (its location is at world coordinate $(a, b)$ with a coordinate rotation of $\theta$ ). Through the detected IR LED signal, both wiimote $3 \mathrm{D}$ pairs will also estimate the positions. With different deployment manner, the localization accuracy is then compared.

Coordinate transformation is a key calculation for such positioning system revolution. 3D position of the infrared LED is sent back to the computer in the coordinate which takes wiimote camera 2 as main reference. Coordinate transformation is later processed using LabVIEW. The transformation is based on the setting of wiimote camera 2, including position $(a, b)$ and the coordinates rotation of $\theta$. The position captured according to the coordinates of wiimote camera 2 is transformed into the position which takes the coordinate of wiimote camera 1 as the main reference. Since the purpose of the development of this system is to improve the capability of this localizing system to adjust to various conditions, multiple choices of settings of wiimote cameras might be available in the same time. As the result, the method to determine the preferred choice with best performance is required. Therefore, the robustness related to the deployment of wiimote camera 2 is discussed. Related robustness simulation is carried out and will be mentioned in detail in the next section.

Further analysis and experiments corresponding to variable-angle dual wiimote $3 \mathrm{D}$ localization is executed for the discussion and demonstration of the performance of this system. To determine the best setup of wiimote cameras, robustness analysis is carried out and discussed separately in angle and position perspective, which are mentioned previously as the main settings of wiimote camera 2 . Besides, the application of this system on robot arm attitude detection is implemented as a practical utilization of this system in indoor positioning purpose. These researches related to variable-angle dual wiimote $3 \mathrm{D}$ localization will be presented in detail in the following sections.

\section{Analysis and Experimental Results}

\subsection{Robustness Analysis}

As mentioned in the previous section, robustness analysis is important for the development of the general indoor localization. Here, robustness of variable-angle dual wiimote $3 \mathrm{D}$ localization is discussed in two geometric variables, the orientation and the position of the deployment, which are the main setting parameters of this system. Simulation is carried out under the assumption that only one variable, either orientation or position, is mistakenly taken into position calculation. Simulation and graphs are performed utilizing LabVIEW. The detailed simulation procedure will be clarified in the following passage.

The overall simulation flow is shown in Figure 7. The simulation is discussed related to two main variables, as mentioned in the previous paragraph, the orientation and position. All setup of both wiimote cameras are assumed to be in the expected condition, except for either the orientation or the position. Since the error in single variable setting is unknown in advance, the parameter used in the calculation of the 3D position of infrared LED is still the expected value. As the result, the final result of positioning appears to be inaccurate. Finally, the error of position in each error magnitude is accumulated and built into a sensitivity curve shown in Figure 8. 


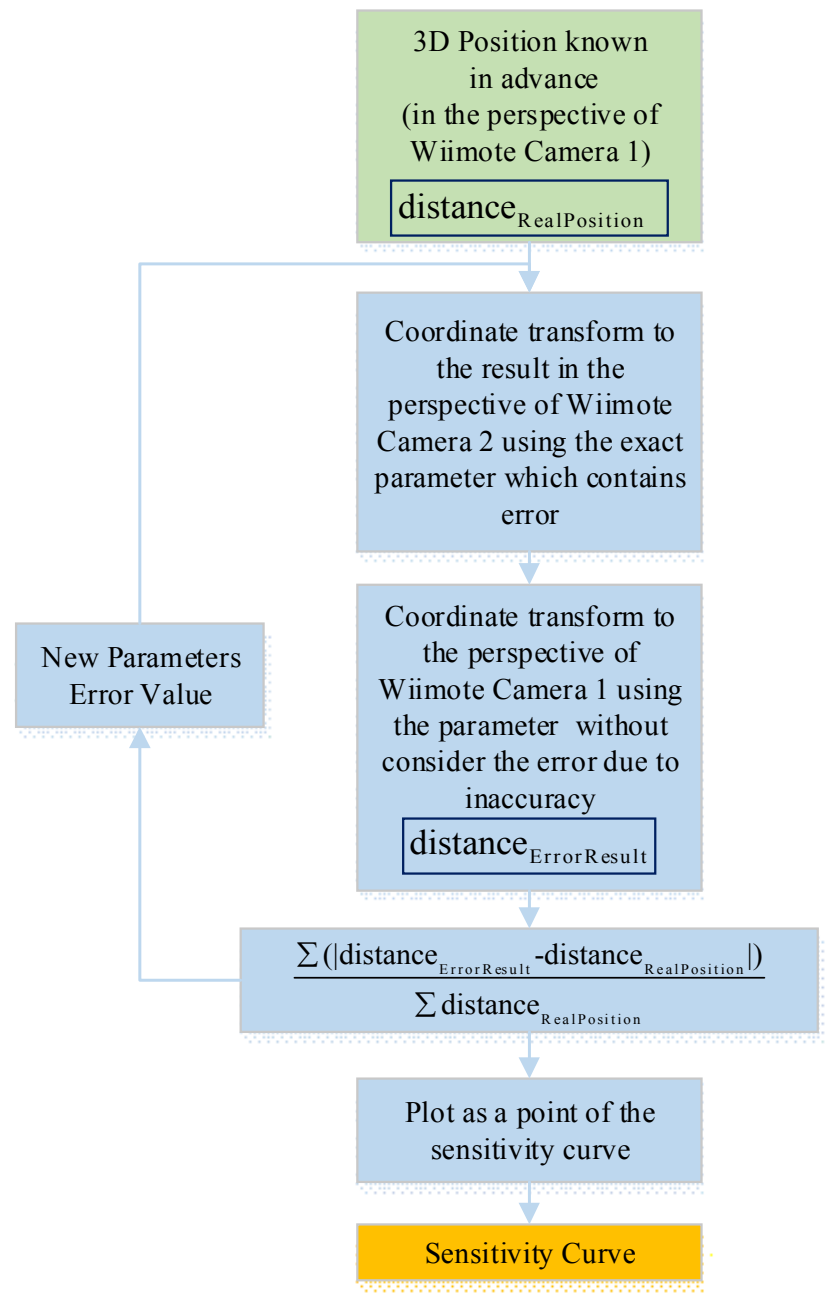

Fig. 7. Process of simulation

Different curves with different basic parameter are placed in the same figure for comparison. The "degree"s shown in legends of Figure 8 indicates the setting of both the expected orientation and the position of wiimote camera 2 . For example, the "60 degree" shown in Figure 8 means wiimote camera 2 is placed in the position $(16.08,30)$, the position where takes $120 \mathrm{~cm}$ as radius and the mid-point as center rotates with the angle of $60^{\circ}$.

As shown in Figure 8(a), when discussing the robustness of the system corresponding to the angle setting, the comparison result shows that the system is less sensitive when the position of wiimote camera 2 is further from $90^{\circ}$ if $2 \%$ is the maximum acceptable error, which implies the setting of wiimote camera 2 should be more accurate in orientation deployment. From Figure 8(b), discussing robustness related to position, the amount of allowed setting inaccuracy in position increases when the setting of wiimote camera 2 is closer to $90^{\circ}$ if $2 \%$ is taken as the limitation of tolerable error. In conclusion, deployment of wiimote camera

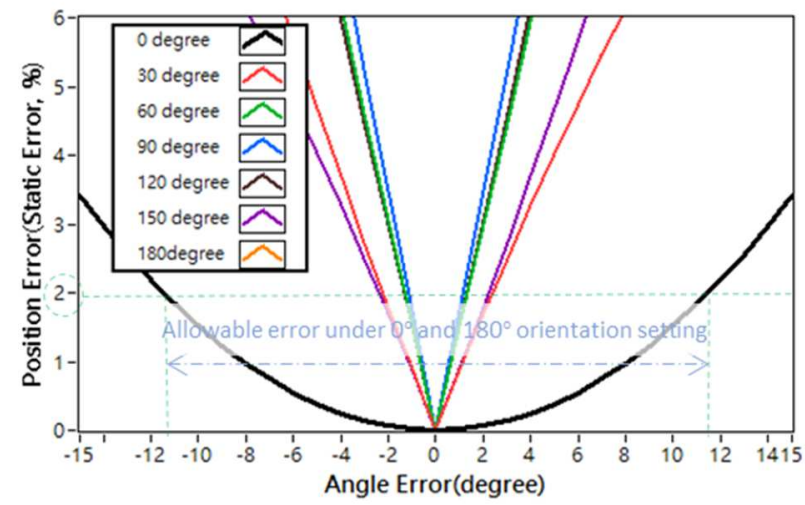

(a) Sensitivity curves when discussing orientation

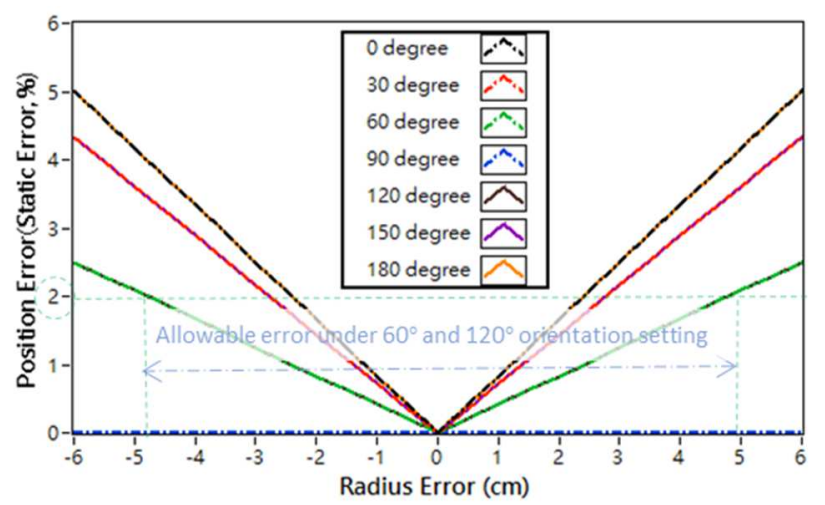

(b) Sensitivity curves when discussing position

Fig. 8. Sensitivity curves of variable-angle dual wiimote 3D localization system

2 should be more precise in orientation when position setting is closer to $90^{\circ}$, and be more accurate in position when the setting is further from $90^{\circ}$.

Corresponding experiment is carried out for this simulation. As shown in Figure 9, IR LED is placed on a 2axis Yokogawa linear servomotor and moves from $(0,80)$ to $(0,160)$. The wiimote cameras are deployed in both " 90 degree" and "135 (-45) degree" cases in order to compare with the result concluded from the simulation. The experimental results, as shown in Figure 10, show similar trend comparing to the simulation result. However, the experimental results show some unideal data, which is obviously influenced by outer factors. For example, the position error corresponding to -9 angle error, as shown in Figure 10(a), is larger than other position error data and is extremely far away from simulation result, and this condition is found happening more than once at the same angle error. Furthermore, position error related to zero angle error or distance error is found occasionally larger than the neighboring data as shown in Figure 10. Possible factors that 
caused such phenomenon is believed to be the change of brightness of IR LED due to different visual angle, and the unexpected light emitted from the environment. From these unexpected experimental results, we found out the effect of deployment on robustness observed from the experimental results is relatively smaller than the effect of outer factors. In the other words, the accuracy of wiimote camera deployment has little effect on the robustness of this system. However, with further improvement in the environmental setting and the stability of the IR LED, the simulation result is believed to play an important role in wiimote camera allocation.

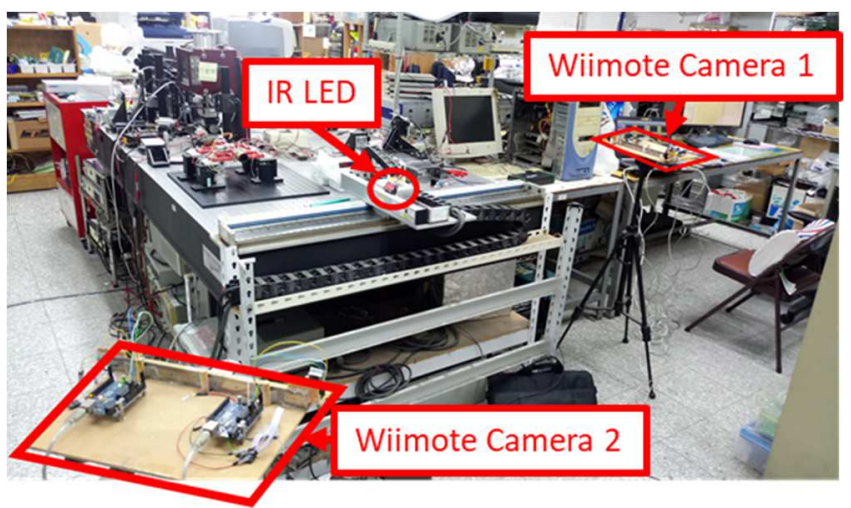

Fig. 9. The experimental setup for robustness analysis

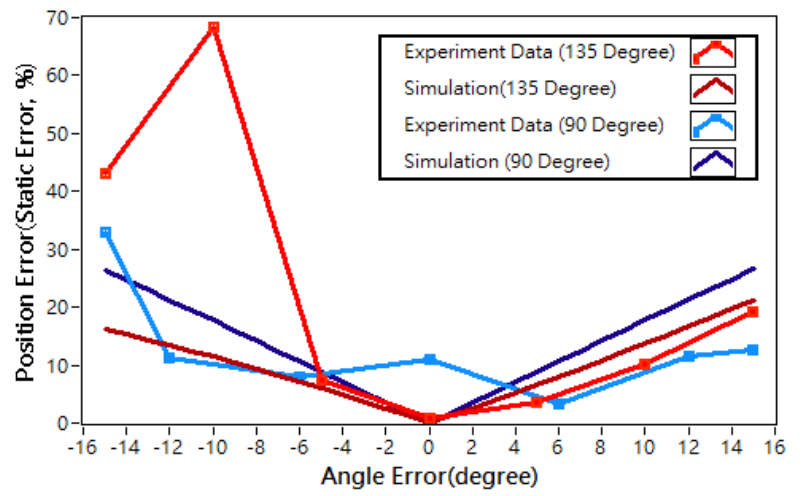

(a) Sensitivity curves when discussing orientation

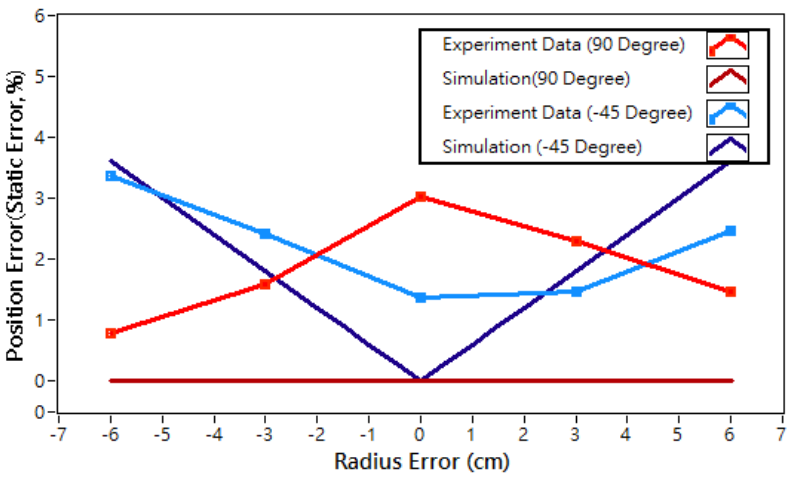

(b) Sensitivity curves when discussing position

\subsection{Robot Arm Attitude Detection}

To demonstrate the effectiveness of wiimote 3D localization system, experiments of robot arm attitude detection is carried out. Two system of wiimote 3D localization system is currently adopted in this experiment, the single wiimote $3 \mathrm{D}$ localization system and IR LED switching technique ${ }^{(7,8)}$.The single wiimote $3 \mathrm{D}$ localization system is applied as the basis of the positioning experiment. The position of the robot arm is captured by the wiimote cameras. Besides, IR LED Switching Technique, the technique developed previously that allows more than 4 LEDs been detected simultaneously ${ }^{(7,8)}$, is applied for the increase of detectable LEDs. As shown in Figure 11, LEDs are located on the ankles of the robot arm and the motion could be successfully expressed in the $3 \mathrm{D}$ graph utilizing LabVIEW.

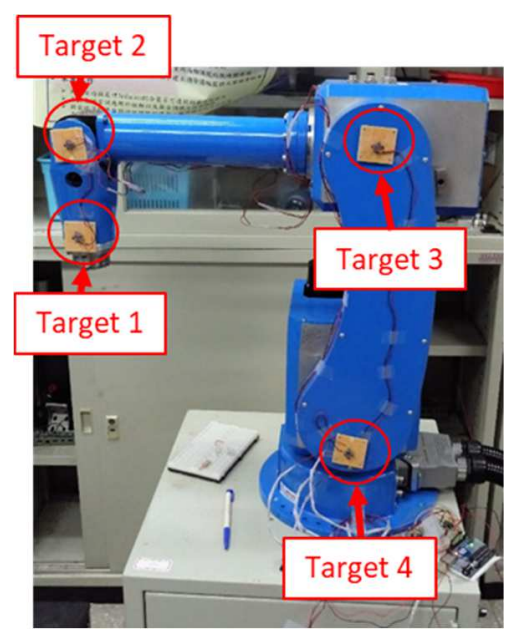

(a) Robot Arm

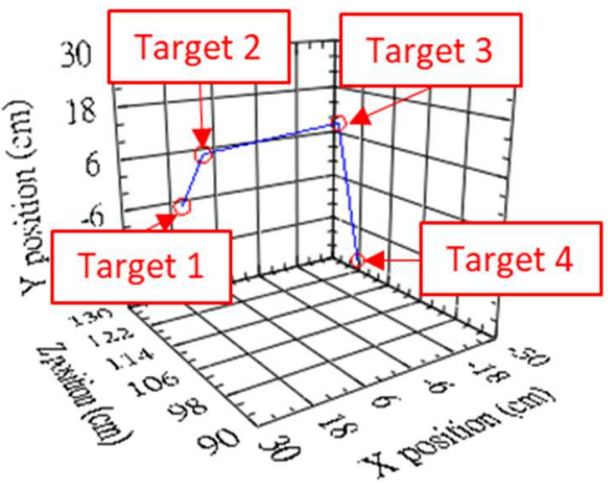

(b) 3D Detection result

Fig. 11. Robot arm attitude detection

Fig. 10. Robustness experimental results 
Since the result of single wiimote 3D localization system has shown as a valid robot arm attitude detection, in the near future, variable-angle wiimote $3 \mathrm{D}$ localization will be applied to the detection of robot arm attitude in order to avoid invisible conditions due to outer obstacles or the changes of attitude of the robot arm. It is believed that with a higher resolution and accuracy, this system is capable to work as an external sensor detecting both the robot arm and the object held by the gripper for the feedback control of robot arm while the current sensor for such control is unable to take the gripped objects into consideration, especially for cases when the objects are flexible and in various shapes.

\section{Discussion and Conclusions}

The development of intelligent living and building automation leads to spotlights on indoor localization. As the result, a series of research on wiimote $3 \mathrm{D}$ localization is executed in order to establish an inexpensive and precise indoor localization system. The Single wiimote 3D localization is initially developed as an application of $3 \mathrm{D}$ positioning utilizing Wii Remote, but is later replaced with fixed-angle wiimote 3D localization system due to the inaccuracy caused by the increase in distance. However, the limitation of the angle deployment is not suitable for the demand of flexibility of allocation corresponding to the various conditions of indoor environments. Hence, variableangle dual wiimote 3D localization system is developed to meet the requirement. Furthermore, robustness analysis is carried out for the determination of the deployment preference. The simulation results show that orientation more when position of wiimote camera 2 is closer to $90^{\circ}$, and position requires more accuracy while wiimote camera 2 is further from $90^{\circ}$. However, the experimental results show that the error on the deployment has little effect on the robustness of this system under current setup condition. In addition, robot arm attitude detection is carried out as a practical application and the result shows this system as a valid positioning approach. In conclusion, with further research on wiimote 3D localization, we believe this simple and effective system has great potential on the establishment of indoor localization system and external sensor for mobile robots.

\section{Acknowledgment}

This work is supported by Ministry of Science and Technology (MOST) of Taiwan under the contract number 107-2813-C-006-117-E and 107-2218-E-006-002-.

\section{References}

(1) S. Vechet, J. Krejsa, P. Houska : "The enhancement of PCSM method by motion history analysis", Recent Advances in Mechatronics, pp. 107-110, Springer, 2007

(2) R. Zhang, F. Höflinger, L. Reindl : "Inertial Sensor Based Indoor Localization and Monitoring System for Emergency Responders", IEEE Sensors Journal, 13, 838-848, 2013

(3) S. J. Kim, B. K. Kim : "Dynamic Ultrasonic Hybrid Localization System for Indoor Mobile Robots", IEEE Trans. Industrial Electronics, 60, 4562-4573, 2013

(4) E. Royer, M. Lhuillier, M. Dhome, J. Lavest : "Monocular Vision for Mobile Robot Localization and Autonomous Navigation", Int. J. Computer Vision, 74, 237-260, 2007

(5) J. Krejsa, S. Vechet : "Infrared Beacons based Localization of Mobile Robot", Electronika ir Electrotechnika, 1, 17-22, 2012

(6) D. Gu, K. S. Chen : "Design and Performance Evaluation of Wiimote-Based Two-Dimensional Indoor Localization Systems for Indoor Mobile Robot Control”, Measurement, 66, 95-108, 2015

(7) T. H. Li, K. S. Chen : "A Wiimote 3D Localization Scheme without Channel Constraints", Mechatronics 2015: Advanced Mechatronics Solutions, pp. 577-582, Springer, 2016

(8) T. H. Li : "Development of 3D Wiimote-Based Localization Scheme and Its Application on Attitude Tracking of Indoor Mobile Robots", Master thesis, National Cheng Kung University, Tainan city, Taiwan, 2015

(9) H. Y. Chan : "Development of 3D Positioning Scheme by Integration of Multiple Wiimote IR Cameras", The $5^{\text {th }}$ IIAE International Conference on Industrial Application Engineering 2017 (ICIAE 2017), pp.30-36, 2017 